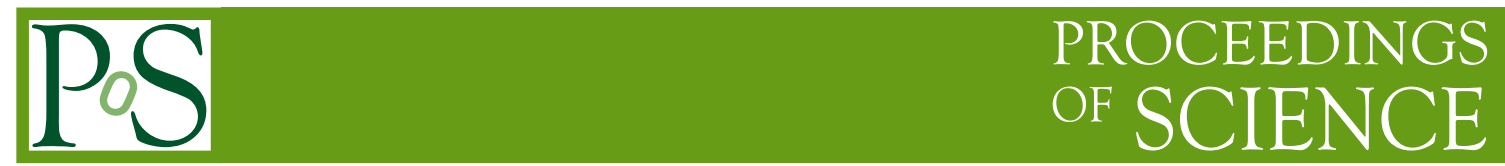

\title{
CP violation in the charm system
}

\section{Angelo Di Canto* \\ Physikalisches Institut, Ruprecht-Karls-Universität Heidelberg, Germany \\ E-mail: angelo.di.canto@cern.ch}

\section{Paride Paradisi}

Theory Division, CERN, Switzerland

E-mail: paride.paradisidcern.ch

\begin{abstract}
We review the experimental status of $\mathrm{CP}$ violation in charm systems and its impact on New Physics scenarios. We emphasize that the synergy of low-energy flavor data with the high- $p_{T}$ part of the LHC program will teach us a lot about new dynamics at the TeV scale (if any) with the upcoming $14 \mathrm{TeV}$ LHC run.
\end{abstract}

VI Italian workshop on p-p physics at the LHC,

8-10 May 2013

Acquario di Genova, Ponte Spinola, Area Porto Antico, Genova, Italy

\footnotetext{
* Speaker.
} 


\section{Introduction}

Processes involving $K$ and $B$ mesons have always been regarded as the most interesting probe of flavor and CP violation. Indeed, within the Standard Model (SM), the largest flavor and CP violating effects reside in systems involving down-type quarks, since the top mass is the main source of flavor violation and charged-current loops are needed to communicate symmetry breaking, in agreement with the GIM mechanism. Similarly, sizable CP violations in the SM are always accompanied by flavor transitions.

While these properties hold in the SM, there is no good reason for them to be true if new physics is present at the electroweak scale. In particular, it is quite plausible that new-physics contributions affect mostly the up-type sector, possibly in association with the mechanism responsible for the large top mass. Supersymmetric models with squark alignment [1,2] provide one example of theories with large flavor and $\mathrm{CP}$ violation in the up sector but, as emphasized also in Ref. $[3,4,5,6]$, this situation is fairly general in classes of models in which the flavor hierarchies are explained without invoking the hypothesis of minimal flavor violation [7]. Hence $D$-meson decays represent a unique probe of new-physics flavor effects, quite complementary to tests in $K$ and $B$ systems.

LHCb has a broad programme of charm physics, which, besides studies of mixing and CP violation, includes searches for rare decays, spectroscopy and measurements of production crosssections. It also has the potential to substantially improve the precision on all the key observables in the charm sector in the next years, thanks to the world's largest, high purity samples of both hadronic and muonic decays already collected during Run I, that will further grow in the coming years of Run II operations. This is possible thanks to the large open charm production crosssection available at the LHC (roughly one tenth of $p p$ collisions produce charm hadrons) and to the excellent particle identification capabilities and the flexible trigger design of the LHCb detector [8].

In the following we present the current status of $C P$ violation in the charm system from both the experimental and theory point of view.

\section{Direct CP violation in singly-Cabibbo-suppressed decays}

Examples of clean, and experimentally favorable channels where to study both direct and indirect $C P$ violation in the charm system are the neutral singly-Cabibbo-suppressed decays into $C P$-eigenstates, such as $D^{0} \rightarrow \pi^{+} \pi^{-}$and $D^{0} \rightarrow K^{+} K^{-}$. Owing to the slow mixing rate of charm mesons, for each decay mode, the $C P$-violating time-integrated asymmetry between decays of states produced as $D^{0}$ and $\bar{D}^{0}$, can be approximated to first order as the sum of two terms [9]:

$$
A_{C P}\left(h^{+} h^{-}\right)=\frac{N\left(D^{0} \rightarrow h^{+} h^{-}\right)-N\left(\bar{D}^{0} \rightarrow h^{+} h^{-}\right)}{N\left(D^{0} \rightarrow h^{+} h^{-}\right)+N\left(\bar{D}^{0} \rightarrow h^{+} h^{-}\right)}=a_{C P}^{\mathrm{dir}}\left(h^{+} h^{-}\right)+\frac{\langle t\rangle}{\tau} a_{C P}^{\mathrm{ind}},
$$

where $h$ identifies a charged kaon or pion. The first term arises from direct $C P$ violation and depends on the decay mode, the second from indirect $C P$ violation, see Eq. (3.2), and is nearly independent of the decay mode [10]. The average decay time of the sample used in the measurement $\langle t\rangle$ depends on the detector acceptance as a function of decay time and $\tau$ is the $D^{0}$ lifetime. To measure each individual asymmetry, the experiments usually determine the number of detected decays of $D^{0}$ and 
$\bar{D}^{0}$ produced in the strong-interaction decay of the $D^{* \pm}$ meson, $D^{*+} \rightarrow D^{0} \pi^{+}$and $D^{*-} \rightarrow \bar{D}^{0} \pi^{-}$, which allows identification of the initial charm flavor through the sign of the charge of the accompanying low-momentum pion. The observed asymmetry is the combination of the contributions from $C P$ violation and from the detection asymmetry between $D^{*+}$ and $D^{*-}$ mesons, due to possibly asymmetric production and different reconstruction efficiency for positive and negative tagging pions. A powerful way to reduce systematic uncertainties induced by these spurious effects is to measure the difference in time-integrated asymmetries in $K^{+} K^{-}$and $\pi^{+} \pi^{-}$final states:

$$
\Delta A_{C P} \equiv A_{C P}\left(K^{+} K^{-}\right)-A_{C P}\left(\pi^{+} \pi^{-}\right) \approx \Delta a_{C P}^{\mathrm{dir}}+\frac{\Delta\langle t\rangle}{\tau} a_{C P}^{\text {ind }},
$$

where $\Delta a_{C P}^{\mathrm{dir}} \equiv a_{C P}^{\mathrm{dir}}\left(K^{+} K^{-}\right)-a_{C P}^{\mathrm{dir}}\left(\pi^{+} \pi^{-}\right)$and the ratio $\Delta\langle t\rangle / \tau$ is equal to zero for the lifetimeunbiased $B$-factory measurements [11, 12], is $0.098 \pm 0.003$ for LHCb [13] and $0.25 \pm 0.04$ for $\mathrm{CDF}$ [9]; therefore $\Delta A_{C P}$ is largely a measure of direct $C P$ violation.

\subsection{Experimental status}

The LHCb collaboration, in late 2011, reported a $3.5 \sigma$ evidence for direct $C P$ violation in twobody, singly-Cabibbo-suppressed $D^{0}$ decays [13] by measuring $\Delta A_{C P}=(-0.82 \pm 0.21$ (stat) \pm 0.11 (syst) $\%$ in a sample of $0.6 \mathrm{fb}^{-1}$ of integrated luminosity. Soon after, the CDF and Belle collaborations presented measurements of $\Delta A_{C P}=(-0.62 \pm 0.21$ (stat) \pm 0.10 (syst) $) \%$ [14] and $\Delta A_{C P}=(-0.87 \pm 0.41$ (stat) \pm 0.06 (syst) $) \%$ [15], respectively. These results, when combined with other measurements of $C P$ violation in singly-Cabibbo-suppressed $D^{0}$ decays, yield as world average $\Delta a_{C P}^{\mathrm{dir}}=(-0.68 \pm 0.15) \%$, which is consistent with no CP violation at $0.002 \%$ CL [16].

Recently the $\mathrm{LHCb}$ collaboration reported an updated preliminary measurement of $\Delta A_{C P}$, based on the $1.0 \mathrm{fb}^{-1}$ sample collected during 2011, which does not confirm the former evidence for CPV in charm [17]:

$$
\Delta A_{C P}=(-0.34 \pm 0.15 \text { (stat) } \pm 0.10(\text { syst })) \% .
$$

This updated analysis, besides the increase in statistics, uses improved detector calibration and vertex reconstruction. The results obtained on the first $0.6 \mathrm{fb}^{-1}$ are consistent within uncertainties between the two analyses. Further, the updated measurement is consistent between the first $0.6 \mathrm{fb}^{-1}$ and the last $0.4 \mathrm{fb}^{-1}$.

In addition, a separated measurement of $\Delta A_{C P}$ has been recently performed by LHCb using $D^{0}$ mesons produced in inclusive semi-muonic $b$-hadron decays, $B \rightarrow D^{0} \mu^{-} X$ and $B \rightarrow \bar{D}^{0} \mu^{+} X$, where the charge of the accompanying muon is used to identify the flavour of the neutral $D$ mesons at production. This analysis [18], which uses the same $1.0 \mathrm{fb}^{-1}$ sample, provides statistically independent information on $\Delta A_{C P}$, being correlation between the two data samples completely negligible, and due to the different production environment and tagging technique is also subject to completely different sources of systematic uncertainties. The measured value of $\Delta A_{C P}$ is found to be $\Delta A_{C P}=(0.49 \pm 0.30$ (stat) \pm 0.14 (syst) $) \%$. This result is compatible at the $3 \% \mathrm{CL}$ with the value measured with $D^{*}$-tagged decays and, if combined with it, yields $\Delta A_{C P}=(-0.15 \pm 0.16) \%$.

The experimental status is summarized in Fig. 1. The new measurements from LHCb do not confirm the evidence of $\mathrm{CP}$ violation in the charm sector that had previously been reported. However, more precise measurements are needed to clarify the experimental picture. The near-future 

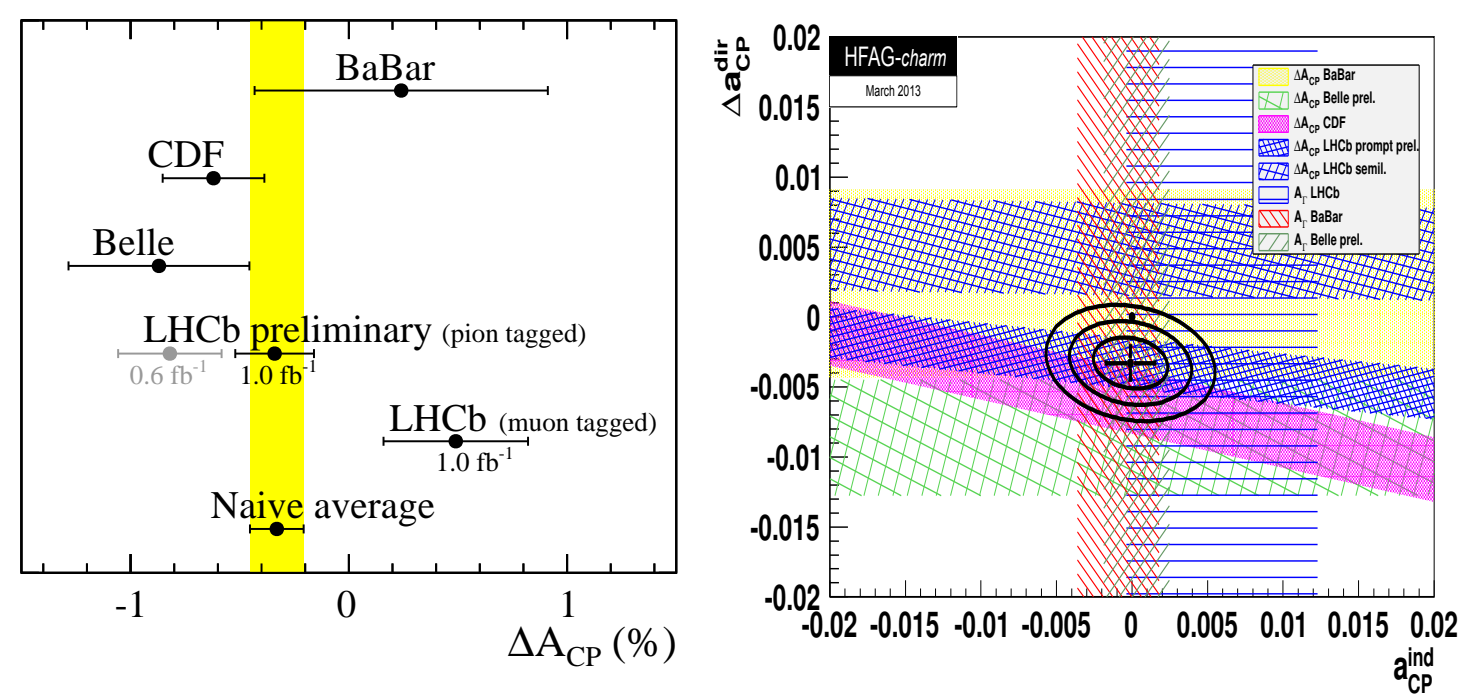

Figure 1: Left plot shows the comparison between available measurements of $\Delta A_{C P}$, with a naive average represented by the yellow band. On the right the combination of all available measurements of CP violation in singly-Cabibbo-suppressed, two-body $D^{0}$ decays as performed by the Heavy Flavor Averaging Group [16] yields $\Delta a_{C P}^{\mathrm{dir}}=(0.33 \pm 0.12) \%, a_{C P}^{\text {ind }}=(-0.01 \pm 0.16) \%$.

term plan is to update the current $\mathrm{LHCb}$ results with the $2.0 \mathrm{fb}^{-1}$ data sample collected during 2012. With this additional statistics and new dedicated analysis, it will also be possible to measure the individual asymmetries, $A_{C P}\left(K^{+} K^{-}\right)$and $A_{C P}\left(\pi^{+} \pi^{-}\right)$, with better precision than currently available best measurements [9]. Other searches for direct $C P$ violation in charged charm decays, as $D_{(s)}^{+} \rightarrow K_{S} h^{+}$and $D_{(s)}^{+} \rightarrow \phi h^{+}$, have already been performed with $\Delta A_{C P}$-style analysis [19] and will soon be updated to the full statistics. No signs of $C P$ violation have been there found.

\subsection{Theory status}

The amount of $C P$ violation in charm decays was generally expected to be much smaller than the $1 \%$ level in the Standard Model [10,20]. This naive expectation was based on the estimates of the "short-distance" penguins with $b$-quarks in the loops, which are suppressed by the combination of CKM elements $V_{c b} V_{u b}^{*} \sim \mathscr{O}\left(\lambda^{5}\right)$, where $\lambda \approx 0.2$ is the Cabibbo angle. The surprisely large, early LHCb result sparked then an intense theoretical debate on whether or not it could be accommodated within the SM. For a comprehensive review see Ref. [21]. We discuss here the impact of physics beyond the Standard Model on $\Delta a_{C P}^{\mathrm{dir}}$, showing its connection with other, non-flavor observables such as nuclear electric dipole moments (EDMs) and flavor-changing neutral current (FCNC) top decays.

On general grounds, models in which the primary source of flavor violation is linked to the breaking of chiral symmetry (left-right flavor mixing) are natural candidates that could introduce sizable direct $C P$-violating effects in charm decays, via enhanced chromomagnetic operators. In the following, we will discuss specific scenarios as supersymmetric models, as well as (nonsupersymmetric) models with Z-mediated and scalar-mediated FCNC. 


\subsubsection{Supersymmetry}

As a first example of explicit new-physics models that can induce an enhanced chromomagnetic operator, we consider the supersymmetric extension of the SM with non-standard sources of flavor symmetry breaking.

The leading contributions stem from loops involving up-squarks and gluinos $[4,10]$. In the mass-insertion approximation and assuming, for illustrative purposes, degenerate supersymmetric masses $\left(\tilde{m}_{q}=\tilde{m}_{g} \equiv \tilde{m}\right)$ one can find

$$
\left|\Delta a_{C P}^{\mathrm{SUSY}}\right| \approx 0.6 \%\left(\frac{\left|\operatorname{Im}\left(\delta_{12}^{u}\right)_{L R}\right|}{10^{-3}}\right)\left(\frac{\mathrm{TeV}}{\tilde{m}}\right) .
$$

In a general supersymmetric framework, we expect the parametric relation

$$
\operatorname{Im}\left(\delta_{12}^{u}\right)_{L R} \approx \frac{\operatorname{Im}(A) \theta_{12} m_{c}}{\tilde{m}} \approx\left(\frac{\operatorname{Im}(A)}{3}\right)\left(\frac{\theta_{12}}{0.3}\right)\left(\frac{\mathrm{TeV}}{\tilde{m}}\right) 0.5 \times 10^{-3},
$$

where $A$ is the trilinear coupling and $\theta_{12}$ is a mixing angle between the first two generations of squarks. From Eq. (2.3) we see that a large (and complex) trilinear coupling $A$, a Cabibbo-size mixing angle, and squarks with $\mathrm{TeV}$ masses give a value of $\operatorname{Im}\left(\delta_{12}^{u}\right)_{L R}$ in the correct ballpark to reproduce the required effect.

While we can envisage scenarios in which flavor violation is restricted to the trilinear terms, it would be fairly unnatural to have this pattern only in the up sector, but not in the down sector. Therefore, if we generalize the structure of Eq. (2.4) to all squarks, we end up with the so-called "disoriented" A-terms scenario [4]

$$
\left(\delta_{i j}^{q}\right)_{L R} \sim \frac{A \theta_{i j}^{q} m_{q_{j}}}{\tilde{m}} \quad q=u, d,
$$

where $\theta_{i j}^{q}$ are generic mixing angles. This pattern amply satisfies all bounds from flavor physics (thanks to the smallness of down-type quark masses) and predicts $\left(\delta_{12}^{u}\right)_{L R} \sim 10^{-3}$. This pattern can be obtained when the matrices of the up and down trilinear coupling constants follow the same hierarchical pattern as the corresponding Yukawa matrices but they do not respect exact proportionality, as it happens in SUSY models with partial compositeness [5].

The most severe suppression in the structure of $\left(\delta_{21}^{u}\right)_{R L}^{\text {eff }}$ shown in Eq. (2.4) is the smallness of the charm mass, or the chirality flip in the second generation. This suppression can be partially avoided by generating the effective 1-2 mixing through the coupling the first two generations to the third one, while taking advantage of the large left-right mixing in the stop sector. This possibility is naturally realized in the supersymmetric framework with split families, where the first two generations of squarks are substantially heavier than $\tilde{t}_{1,2}$ and $\tilde{b}_{L}$, the only squarks required to be close to the electroweak scale by naturalness arguments.

Within this framework we can decompose the effective couplings relevant to $\Delta a_{C P}^{\mathrm{SUSY}}$ as follows

$$
\begin{aligned}
& \left(\delta_{12}^{u}\right)_{R L}^{\mathrm{eff}}=\left(\delta_{13}^{u}\right)_{R R}\left(\delta_{33}^{u}\right)_{R L}\left(\delta_{32}^{u}\right)_{L L} \\
& \left(\delta_{12}^{u}\right)_{L R}^{\mathrm{eff}}=\left(\delta_{13}^{u}\right)_{L L}\left(\delta_{33}^{u}\right)_{R L}\left(\delta_{32}^{u}\right)_{R R}
\end{aligned}
$$

This decomposition allows us to draw the following general considerations. 
LR mixing: Since $\left(\delta_{33}^{u}\right)_{R L}$ in the stop sector can be approximately equal to one, it does not represent a significant suppression factor. Note that a Higgs mass around $125 \mathrm{GeV}$, naturally favors a large $A$ term if we want to keep the stop below $1 \mathrm{TeV}$. Thus the recent Higgs data support the assumption that $\left(\delta_{33}^{u}\right)_{R L}$ is of order unity.

RR mixing: The mixing $\left(\delta_{3 i}^{u}\right)_{R R}$, for $i=1,2$ in the up-type right-handed sector is relatively unconstrained. The only significant bound comes from $D-\bar{D}$ mixing, which implies $\mid\left(\delta_{31}^{u}\right)_{R R} \times$ $\left(\delta_{32}^{u}\right)_{R R} \mid \lesssim 10^{-2}$ for squark masses of $1 \mathrm{TeV}$.

LL mixing: The off-diagonal elements of the CKM matrix provide natural reference values for the mixing in the left-handed sector, namely $\left|\left(\delta_{3 i}^{u}\right)_{L L}\right|=O\left(\left|V_{t i}\right|\right)$.

In particular, we can consider the following two options to explain the $\mathrm{LHCb}$ results:

$$
\begin{gathered}
\left(\delta_{32}^{u}\right)_{L L}=O\left(\lambda^{2}\right), \quad\left(\delta_{13}^{u}\right)_{R R}=O\left(\lambda^{2}\right) \\
\left(\delta_{13}^{u}\right)_{L L}=O\left(\lambda^{3}\right), \quad\left(\delta_{32}^{u}\right)_{R R}=O(\lambda) .
\end{gathered}
$$

Both solutions can be simultaneously operative as they lead to $\left(\delta_{12}^{u}\right)_{R L}^{\text {eff }}=O\left(\lambda^{4}\right)=O\left(10^{-3}\right)$ and $\left(\delta_{12}^{u}\right)_{L R}^{\text {eff }}=O\left(\lambda^{4}\right)=O\left(10^{-3}\right)$, respectively.

The presence of new $\mathrm{CP}$ violating phases are expected to generate hadronic electric dipole moments. Gluino-squark loops, analogous to the one inducing the $\Delta C=1$ chromomagnetic operator, yield an $\operatorname{EDM}\left(d_{u}\right)$ and a chromo-EDM $\left(d_{u}^{c}\right)$ for the up quark. Among the hadronic EDMs, the best constraints come from mercury and neutron EDMs ( $d_{\mathrm{Hg}}$ and $d_{n}$ respectively). The current experimental bounds read $\left|d_{n}\right|<2.9 \times 10^{-26} e \mathrm{~cm}\left(90 \% \mathrm{CL}\right.$ ) [22] and $\left|d_{\mathrm{Hg}}\right|<3.1 \times 10^{-29} \mathrm{ecm}$ (95\% CL) [23].

Assuming degenerate supersymmetric masses one can find for instance that

$$
\left|d_{n}\right| \approx 3 \times 10^{-26}\left(\frac{\left|\operatorname{Im}\left(\delta_{11}^{u}\right)_{L R}\right|}{10^{-6}}\right)\left(\frac{\mathrm{TeV}}{\tilde{m}}\right) e \mathrm{~cm},
$$

and therefore it has to be seen whether concrete SUSY scenario can naturally account for the required suppression $\left|\operatorname{Im}\left(\delta_{11}^{u}\right)_{L R}\right| \lesssim 10^{-6}$. Similar conclusions arise in the split-family case.

\subsubsection{New-physics scenarios with Z-mediated FCNC}

Effective FCNC couplings of the $Z$ boson to SM quarks, or between quarks and heavier fermions, can appear in several new-physics frameworks. Prominent examples are the SM with non-sequential generations of quarks, models with an extra $U(1)$ symmetry or models with extra vector-like doublets and singlets.

Irrespective of the underlying dynamics, we introduce the following effective Lagrangian to describe the FCNC couplings of the $Z$-boson to fermions

$$
\mathscr{L}_{\mathrm{eff}}^{Z}=-\frac{g}{2 \cos \theta_{W}} \bar{F}_{i} \gamma^{\mu}\left[\left(g_{L}^{Z}\right)_{i j} P_{L}+\left(g_{R}^{Z}\right)_{i j} P_{R}\right] q_{j} Z_{\mu}+\text { h.c. }
$$

where $g$ is the $S U(2)_{L}$ gauge coupling, and $F$ can be either a SM quark $(F=q)$ or some heavier non-standard fermion. 


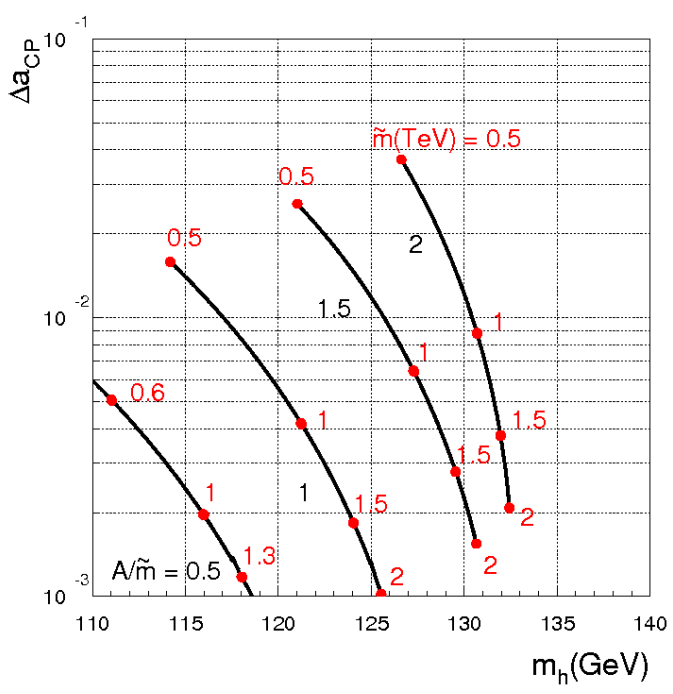

Figure 2: $\Delta a_{C P}^{\text {SUSY }}$ vs. $m_{h}$ for $\left|\operatorname{Im}\left[\left(\delta_{32}^{u}\right)_{R R}\left(\delta_{31}^{u}\right)_{L L}\right]\right|=10^{-2}, \tilde{m} \leq 2 \mathrm{TeV}$, and $A=0.5,1,1.5,2$ from Ref. [4].

The chromomagnetic operator is generated at the one-loop level, with leading contribution from Z-top exchange diagrams leading to [4]

$$
\left|\Delta a_{C P}^{Z-\mathrm{FCNC}}\right| \approx 0.6 \%\left|\frac{\operatorname{Im}\left[\left(g_{L}^{Z}\right)_{u t}^{*}\left(g_{R}^{Z}\right)_{c t}\right]}{2 \times 10^{-4}}\right| .
$$

Strong constraints on the $\left(g_{L, R}^{Z}\right)_{i j}$ effective couplings for up-type quarks arise from $D^{0}-\bar{D}^{0}$ mixing: from tree-level $Z$ exchange diagrams one gets $\left|\left(g_{L, R}^{Z}\right)_{u c}\right|<2 \times 10^{-4}$ and $\left|\left(g_{L}^{Z}\right)_{u c}\left(g_{R}^{Z}\right)_{u c}\right|<0.5 \times$ $10^{-8}$. However, much weaker constraints are derived on the effective couplings involving the top, appearing in Eq. (2.11), since they contribute to $D^{0}-\bar{D}^{0}$ mixing only at the one-loop level.

The presence of new $\mathrm{CP}$ violating phases in the couplings $\left(g_{L, R}^{Z}\right)_{i j}$ are expected to generate also hadronic EDMs, with a strong correlation to $\Delta a_{C P}$. In particular, one can find [4]

$$
\left|d_{n}\right| \approx 3 \times 10^{-26}\left|\frac{\operatorname{Im}\left[\left(g_{L}^{Z}\right)_{u t}^{*}\left(g_{R}^{Z}\right)_{u t}\right]}{2 \times 10^{-7}}\right| e \mathrm{~cm} .
$$

Comparing the above result with Eq. (2.11) we find that a contribution to $\Delta a_{C P}$ at the per-cent level is allowed only if there exists a strong hierarchy among the $\left(g_{L, R}^{Z}\right)_{t q}$ couplings.

In the NP scenarios with Z-mediated FCNCs, the most interesting FCNC processes in the top sector are $t \rightarrow c Z$ and $t \rightarrow u Z$, which arise at the tree level. In particular, it turns out that [4]

$$
\operatorname{Br}(t \rightarrow c Z) \approx 0.7 \times 10^{-2}\left|\frac{\left(g_{R}^{Z}\right)_{t c}}{10^{-1}}\right|^{2},
$$

which is within the reach of the LHC for the values of $\left(g_{R}^{Z}\right)_{t c}$ relevant to $\Delta a_{C P}^{Z-F C N C}$.

\subsubsection{New-physics scenarios with scalar-mediated FCNC}

We finally analyze a new-physics framework with effective FCNC couplings to SM quarks of a scalar particle, which can be either the SM Higgs or some new scalar state. In analogy to 
Eq. (2.10) we introduce the following effective Lagrangian

$$
\mathscr{L}_{\text {eff }}^{h}=-\bar{q}_{i}\left[\left(g_{L}^{h}\right)_{i j} P_{L}+\left(g_{R}^{h}\right)_{i j} P_{R}\right] q_{j} h+\text { h.c. },
$$

where $h$ is the scalar state. The scalar field $h$ could be identified with the physical Higgs boson, for instance in models with non-renormalizable interactions between quarks and multiple powers of the Higgs field.

Also in this case the chromomagnetic operator is generated at the one-loop level, with a leading contribution from $h$-top exchange diagrams. This leads to [4]

$$
\left|\Delta a_{C P}^{h-\mathrm{FCNC}}\right| \approx 0.6 \%\left|\frac{\operatorname{Im}\left[\left(g_{L}^{h}\right)_{u t}^{*}\left(g_{R}^{h}\right)_{t c}\right]}{2 \times 10^{-4}}\right| .
$$

where we have assumed $m_{h}=125 \mathrm{GeV}$. For the hadronic EDMs it turns out that [4]

$$
\left|d_{n}\right| \approx 3 \times 10^{-26}\left|\frac{\operatorname{Im}\left[\left(g_{L}^{h}\right)_{u t}^{*}\left(g_{R}^{h}\right)_{t u}\right]}{2 \times 10^{-7}}\right| e \mathrm{~cm} .
$$

With scalar-mediated FCNCs, the potentially most interesting signal are the rare top decays $t \rightarrow c h$ or $t \rightarrow u h$. In particular, one can find that [4]

$$
\operatorname{Br}(t \rightarrow q h) \approx 0.4 \times 10^{-2}\left|\frac{\left(g_{R}^{h}\right)^{t q}}{10^{-1}}\right|^{2}
$$

which could be within the reach of the LHC.

The discussion about the bounds from $D^{0}-\bar{D}^{0}$ and $B_{s, d}-\bar{B}_{s, d}$ mixing proceeds in a similar way to the $Z$ FCNC case.

In order to establish whether the observed $\Delta a_{C P}$ can be accommodated in the SM or not, it would be important to monitor other observables which are sensitive to the same (potential) NP effect. In NP scenarios where $\Delta a_{C P}$ mostly arises from the chromomagnetic operator, the direct CP asymmetries in radiative decays $D \rightarrow P^{+} P^{-} \gamma(P=\pi, K)$ are the best candidates to make such a test, as recently pointed out in Ref. [24]. Indeed, even if $D \rightarrow P^{+} P^{-} \gamma$ is generated by the electromagnetic operator, many NP scenarios predict comparable effects for the chromomagnetic and electromagnetic operators. In any case, a non-vanishing chromomagnetic operator at the high scale unavoidably contributes to the electromagnetic operator through QCD running effects.

\section{3. $C P$ violation in $D^{0}-\bar{D}^{0}$ mixing}

The mass eigenstates of neutral $D$ mesons, $\left|D_{1,2}\right\rangle$, with masses $m_{1,2}$ and widths $\Gamma_{1,2}$ can be written as linear combinations of the flavour eigenstates $\left|D_{1,2}\right\rangle=p\left|D^{0}\right\rangle \pm q\left|\bar{D}^{0}\right\rangle$, with complex coefficients $p$ and $q$ which satisfy $|p|^{2}+|q|^{2}=1$. The average mass and width are defined as $m \equiv\left(m_{1}+m_{2}\right) / 2$ and $\Gamma \equiv\left(\Gamma_{1}+\Gamma_{2}\right) / 2$. The $D$ mixing parameters are defined using the mass and width difference as $x \equiv\left(m_{2}-m_{1}\right) / \Gamma$ and $y \equiv\left(\Gamma_{2}-\Gamma_{1}\right) / 2 \Gamma$. The phase convention of $p$ and $q$ is chosen such that $C P\left|D^{0}\right\rangle=-\left|\bar{D}^{0}\right\rangle$. CP is conserved if $|q / p|=1$ and $\phi=\arg (q / p)=0$.

First evidence for mixing of neutral $D^{0}$ mesons was discovered in 2007 by $B$-factories $[25,26]$ and is now well established [16]: the no-mixing hypothesis is excluded at more than $10 \sigma$ for the 

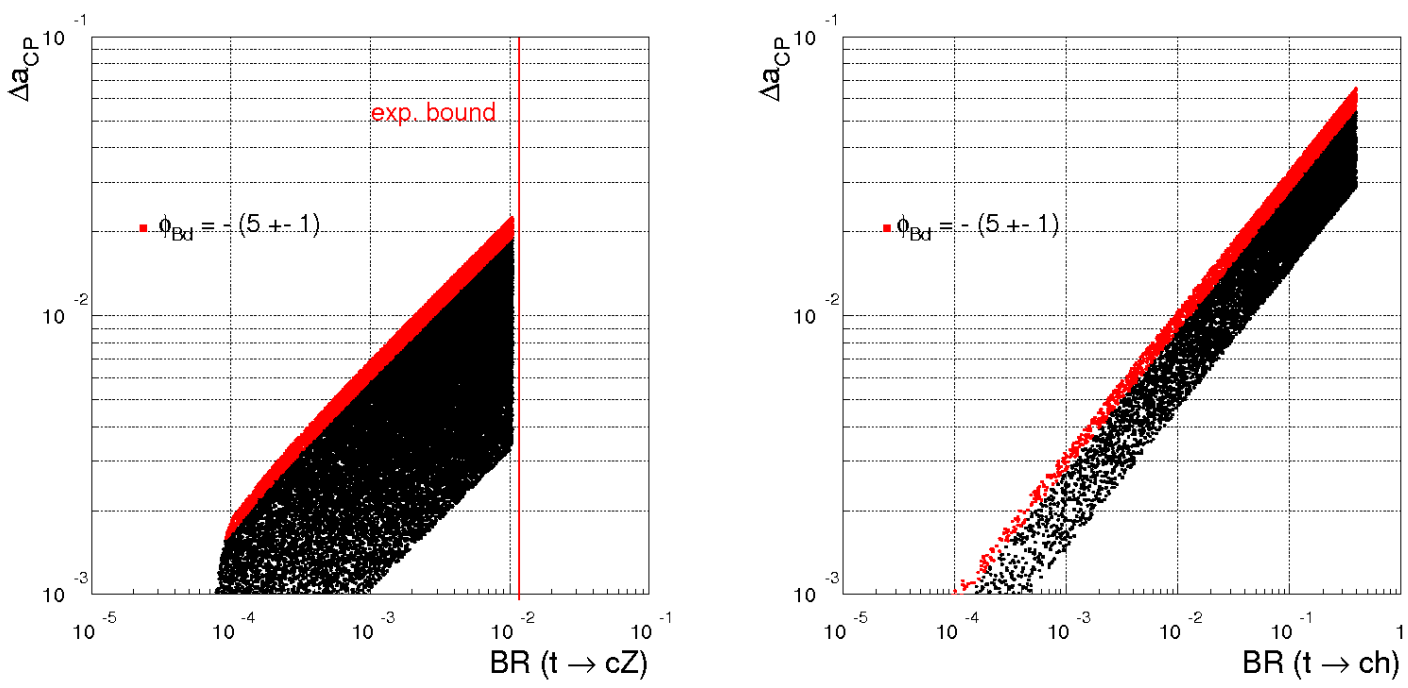

Figure 3: Left: $\mathrm{BR}(t \rightarrow c Z)$ vs. $\Delta a_{C P}^{Z-\mathrm{FCNC}}$. Right: $\mathrm{BR}(t \rightarrow c h)$ vs. $\Delta a_{C P}^{h-\mathrm{FCNC}}$ from Ref. [4]. The plots have been obtained by means of the scan: $\left|\left(g_{L}^{X}\right)_{u t}\right|>10^{-3},\left|\left(g_{R}^{X}\right)_{c t}\right|>10^{-2}$, where $X=Z$, h, with $\arg \left[\left(g_{L}^{X}\right)_{u t}\right]=$ $\pm \pi / 4$ and $\arg \left[\left(g_{R}^{X}\right)_{c t}\right]=0$. The points in the red regions solve the tension in the CKM fits through a nonstandard phase in $B_{d}-\bar{B}_{d}$ mixing.

world average $\left(x=\left(0.49_{-0.18}^{+0.17}\right) \%, y=(0.74 \pm 0.09) \%\right)$ and no evidence of indirect $C P$ violation has yet been found $\left(|q / p| \in[0.44,1.07], \phi \in\left[-44.6^{\circ},-7.5^{\circ}\right]\right.$ at $\left.95 \% \mathrm{CL}\right)$. Recently, LHCb presented the first observation of charm mixing from a single measurement [27]. The analysis uses the $1.0 \mathrm{fb}^{-1}$ sample collected during 2011 and shows the potentiality for more precise results to be available already before the beginning of Run II.

The observed mixing rate is consistent with, but at the upper end of, SM expectations [28] and constrains many NP models [29]. It is interesting to notice that model independent correlations between different $C P$-violating observables also help in further constrain the underlying dynamic.

As an example, it can be shown that there exists a model independent relation between $x, y$, $|q / p|$ and $\phi$ which implies the following correlation [30, 31, 32] (see Fig. 4)

$$
A_{\Gamma}=\frac{x^{2}+y^{2}}{4|y|} a_{\mathrm{SL}}
$$

between two widely used experimental observables: the asymmetry between the effective lifetimes of $D^{0}$ decays to $C P$ eigenstates,

$$
\begin{aligned}
A_{\Gamma} & =\frac{\hat{\tau}\left(\bar{D}^{0} \rightarrow h^{+} h^{-}\right)-\hat{\tau}\left(D^{0} \rightarrow h^{+} h^{-}\right)}{\hat{\tau}\left(\bar{D}^{0} \rightarrow h^{+} h^{-}\right)+\hat{\tau}\left(\bar{D}^{0} \rightarrow h^{+} h^{-}\right)} \\
& =-a_{C P}^{\text {ind }} \approx \frac{y}{2}\left(\left|\frac{q}{p}\right|-\left|\frac{p}{q}\right|\right) \cos \phi-\frac{x}{2}\left(\left|\frac{q}{p}\right|+\left|\frac{p}{q}\right|\right) \sin \phi,
\end{aligned}
$$

and the semileptonic asymmetry in the decay to "wrong sign" leptons,

$$
a_{\mathrm{SL}}=\frac{\Gamma\left(D^{0} \rightarrow h^{+} \ell^{-} v\right)-\Gamma\left(\bar{D}^{0} \rightarrow h^{-} \ell^{+} v\right)}{\Gamma\left(D^{0} \rightarrow h^{+} \ell^{-} v\right)+\Gamma\left(\bar{D}^{0} \rightarrow h^{-} \ell^{+} v\right)}=\frac{|q|^{4}-|p|^{4}}{|q|^{4}+|p|^{4}}
$$

which is a direct measure of $C P$ violation in mixing. 

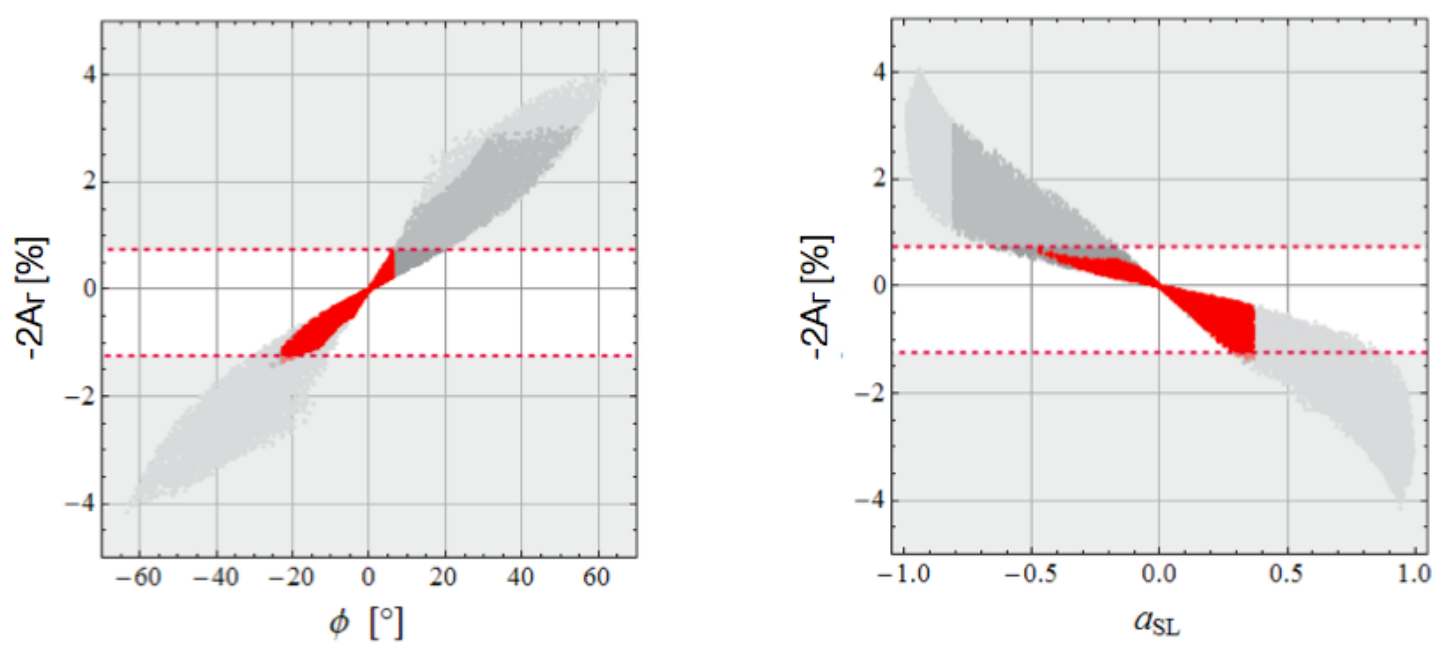

Figure 4: Model independent correlations between $A_{\Gamma}$ and $\phi$ (left) or $a_{\mathrm{SL}}$ (right) from Ref. [33]. Light gray satisfies $x \in[0.46,1.46] \%$ and $y \in[0.51,1.15] \%$; darker gray further satisfies $|q / p| \in[0.57,1.21]$; red is compatible with all above constraints plus $\phi \in\left[-22.5^{\circ}, 6.3^{\circ}\right]$; the dashed lines stand for the resulting allowed range for $A_{\Gamma}$.

\section{Conclusions}

It is quite plausible that new physics contributions affect mostly the up sector $[3,4,5,6]$, hence $C P /$ flavor violation in $D$ mesons decays provides a unique probe of beyond SM flavor effects, which is quite complementary to tests in $K$ and $B$ systems and has not yet been completely exploited by the experimentalists. The early evidence for large direct $C P$ violation in charm by $\mathrm{LHCb}$, even if recently not confirmed, has stimulated new ideas in the theory community and the construction of models departing in a controlled way from the MFV paradigm $[4,5,6]$. These models have a much broader impact on low and high- $p_{T}$ phenomenology, which is hopefully testable at the LHC. The full LHCb Run I dataset has still to be analyzed, then additional investigations of the charm sector with more precise results will help constraining new physics model already before the beginning of Run II. The synergy of low-energy flavor data with the high- $p_{T}$ part of the LHC program will teach us a lot about new dynamics at the TeV scale (if any) with the upcoming $14 \mathrm{TeV}$ LHC run.

\section{References}

[1] Y. Nir and N. Seiberg, Phys. Lett. B 309 (1993) 337.

[2] M. Leurer, Y. Nir and N. Seiberg, Nucl. Phys. B 420 (1994) 468.

[3] G. F. Giudice, B. Gripaios and R. Sundrum, JHEP 1108 (2011) 055.

[4] G. F. Giudice, G. Isidori and P. Paradisi, JHEP 1204 (2012) 060.

[5] B. Keren-Zur, P. Lodone, M. Nardecchia, D. Pappadopulo, R. Rattazzi and L. Vecchi, Nucl. Phys. B 867, 429 (2013).

[6] L. Calibbi, P. Paradisi and R. Ziegler, JHEP 1306 (2013) 052. 
[7] G. D’Ambrosio, G. F. Giudice, G. Isidori and A. Strumia, Nucl. Phys. B 645 (2002) 155 [hep-ph/0207036].

[8] A. A. Alves Jr. et al. [LHCb Collaboration], JINST 3 (2008) S08005.

[9] T. Aaltonen et al. [CDF Collaboration], Phys. Rev. D 85 (2012) 012009.

[10] Y. Grossman, A. L. Kagan and Y. Nir, Phys. Rev. D 75 (2007) 036008.

[11] B. Aubert et al. [BaBar Collaboration], Phys. Rev. Lett. 100 (2008) 061803.

[12] M. Staric et al. [Belle Collaboration], Phys. Lett. B 670 (2008) 190.

[13] R. Aaij et al. [LHCb Collaboration], Phys. Rev. Lett. 108 (2012) 111602.

[14] T. Aaltonen et al. [CDF Collaboration], Phys. Rev. Lett. 109 (2012) 111801.

[15] B. R. Ko [Belle Collaboration], arXiv:1212.1975 [hep-ex].

[16] Y. Amhis et al. (Heavy Flavour Averaging Group), arXiv:1207.1158 and online updates at http: //www.slac.stanford.edu/xorg/hfag.

[17] R. Aaji et al. [LHCb Collaboration], LHCb-CONF-2013-003.

[18] R. Aaij et al. [LHCb Collaboration], Phys. Lett. B 723 (2013) 33.

[19] R. Aaij et al. [ LHCb Collaboration], arXiv:1303.4906 [hep-ex].

[20] S. Bianco, F. L. Fabbri, D. Benson and I. Bigi, Riv. Nuovo Cim. 26N7 (2003) 1.

[21] R. Aaij et al. [LHCb Collaboration], Eur. Phys. J. C 73 (2013) 2373.

[22] C. A. Baker et al., Phys. Rev. Lett. 97 (2006) 131801. [arXiv:hep-ex/0602020].

[23] W. C. Griffith, M. D. Swallows, T. H. Loftus, M. V. Romalis, B. R. Heckel and E. N. Fortson, Phys. Rev. Lett. 102 (2009) 101601.

[24] G. Isidori and J. F. Kamenik, Phys. Rev. Lett. 109 (2012) 171801 [arXiv:1205.3164 [hep-ph]].

[25] B. Aubert et al. [BaBar Collaboration], Phys. Rev. Lett. 98 (2007) 211802.

[26] M. Staric et al. [Belle Collaboration], Phys. Rev. Lett. 98 (2007) 211803.

[27] R. Aaij et al. [LHCb Collaboration], Phys. Rev. Lett. 110 (2013) 101802.

[28] A. F. Falk, Y. Grossman, Z. Ligeti, Y. Nir and A. A. Petrov, Phys. Rev. D 69 (2004) 114021.

[29] E. Golowich, J. Hewett, S. Pakvasa and A. A. Petrov, Phys. Rev. D 76 (2007) 095009.

[30] Y. Grossman, Y. Nir and G. Perez, Phys. Rev. Lett. 103 (2009) 071602.

[31] I. I. Bigi, M. Blanke, A. J. Buras and S. Recksiegel, JHEP 0907 (2009) 097.

[32] A. L. Kagan and M. D. Sokoloff, Phys. Rev. D 80 (2009) 076008.

[33] W. Altmannshofer, A. J. Buras and P. Paradisi, Phys. Lett. B 688 (2010) 202. 